\title{
Schatzker Type VI Injury with Missed Posterior Dislocation of the Hip
}

\author{
Murali Mothilal ${ }^{1}$, F Abdul Khader ${ }^{2}$, T Sundara Rajan ${ }^{3}$, Jamal Mohammad ${ }^{4}$
}

\section{Abstract}

Introduction: Posterior dislocation of the hip can be an associated injury with fracture neck and shaft of the femur fractures.

Case description: A 33-year-old male came to us with above knee slab of the left leg. He had a fall from the bike and took initial treatment elsewhere. The X-ray and diagnosis of Schatzker type VI injury of the left knee were already done in the previous hospital. The upper tibia fixed with L-plate with screws. When the patient started walking in parallel bar, he felt twisting strain on the left hip and complained of pain in the left hip region. Now, the patient had shortening and internal rotation of the left lower limb. Clinically diagnosed posterior dislocation of the left hip was confirmed with X-ray. Reduction of dislocation was done.

Conclusion: $\mathrm{X}$-rays of proximal and distal joints are mandatory. The statement stands testimony every time.

Keywords: Posterior dislocation of the hip, Schatzker type VI injury, Tibial plateau fractures.

Journal of Orthopedics and Joint Surgery (2020): 10.5005/jp-journals-10079-1017
\end{abstract}

\section{INTRODUCTION}

Tibial plateau fractures account for $1 \%$ of all fractures and are typically sustained with high-energy mechanisms. ${ }^{1}$ These fractures can be associated with other injuries like meniscal tear, ligamentous injuries, compartmental syndrome, and fractures of the patella.

There is no mention about associated ipsilateral posterior dislocation of the hip (associated tibial plateau fractures) in the literature reviewed. Posterior dislocation of the hip can be an associated injury with fracture neck of femur and shaft of the femur usually. Posterior dislocation of the hip associated with ipsilateral knee fractures is common, especially patellar fractures, open knee lacerations, and ligamentous injury. ${ }^{2}$

We are herewith reporting a case of Schatzker type VI fracture associated with posterior dislocation of the hip.

\section{Case Description}

A 33-year-old male came to our hospital on August 12, 2014, with above knee slab of the left leg. He had a fall from the bike on that day. He was not able to describe the exact mechanism of the injury, but he found that his bike was lying on his knee region. He had initial treatment elsewhere, and he came to us with above knee slab on the left leg with a diagnosis of Schatzker type VI injury of the left knee (Fig. 1).

The slab was opened and examined. There were two wounds of $2 \mathrm{~cm}$ each just below the left knee region and a large abrasion of $5 \times 2 \mathrm{~cm}$ over the medial aspect of the left knee. CT knee showed fracture of lateral femoral condyle and fracture of patella of the left knee in addition. The fracture of upper tibia fixed with L-plate with screws and lateral femoral condyle with cancellous screws (Fig. 2).

Two weeks after surgery, the patient was mobilized and started walking in parallel bar. While walking, he felt twisting strain on the affected leg and complained of pain in the left hip region. Now, when we examined the patient, he had shortening and internal rotation of the left lower limb. Clinical diagnosis of posterior dislocation of the left hip was suspected, and X-ray was taken. X-ray showed posteriorly dislocated left hip (Fig. 3).
${ }^{1}$ Department of Orthopedics, Sri Muthukumaran Medical College Hospital, Chennai, Tamil Nadu, India

${ }^{2,3}$ Department of Orthopedics, Shri Sathya Sai Medical College and Research Institute, Chennai, Tamil Nadu, India

${ }^{4}$ Department of Orthopedics, Chettinad Medical College, Chennai, Tamil Nadu, India

Corresponding Author: Murali Mothilal, Department of Orthopedics, Sri Muthukumaran Medical College Hospital, Chennai, Tamil Nadu, India, Phone: +91 9445373395, e-mail: muraliorth@gmail.com

How to cite this article: Mothilal M, Khader FA, Rajan TS, et al. Schatzker Type VI Injury with Missed Posterior Dislocation of the Hip. J Orth Joint Surg 2020;2(1):43-45.

Source of support: Nil

Conflict of interest: None

We presumed that the dislocation had occurred at the time of the accident and not because of twisting strain in the parallel bar walking. The patient did not complain of any pain in the hip region

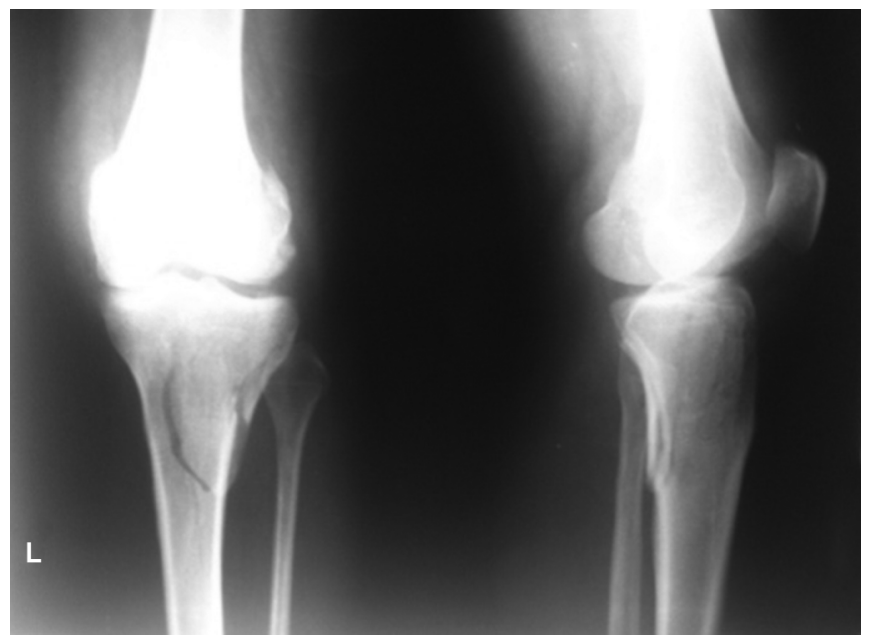

Fig. 1: X-ray showing fracture of Schatzker type VI injury of the left tibia

(0) The Author(s). 2020 Open Access This article is distributed under the terms of the Creative Commons Attribution 4.0 International License (https://creativecommons. org/licenses/by-nc/4.0/), which permits unrestricted use, distribution, and non-commercial reproduction in any medium, provided you give appropriate credit to the original author(s) and the source, provide a link to the Creative Commons license, and indicate if changes were made. The Creative Commons Public Domain Dedication waiver (http://creativecommons.org/publicdomain/zero/1.0/) applies to the data made available in this article, unless otherwise stated. 

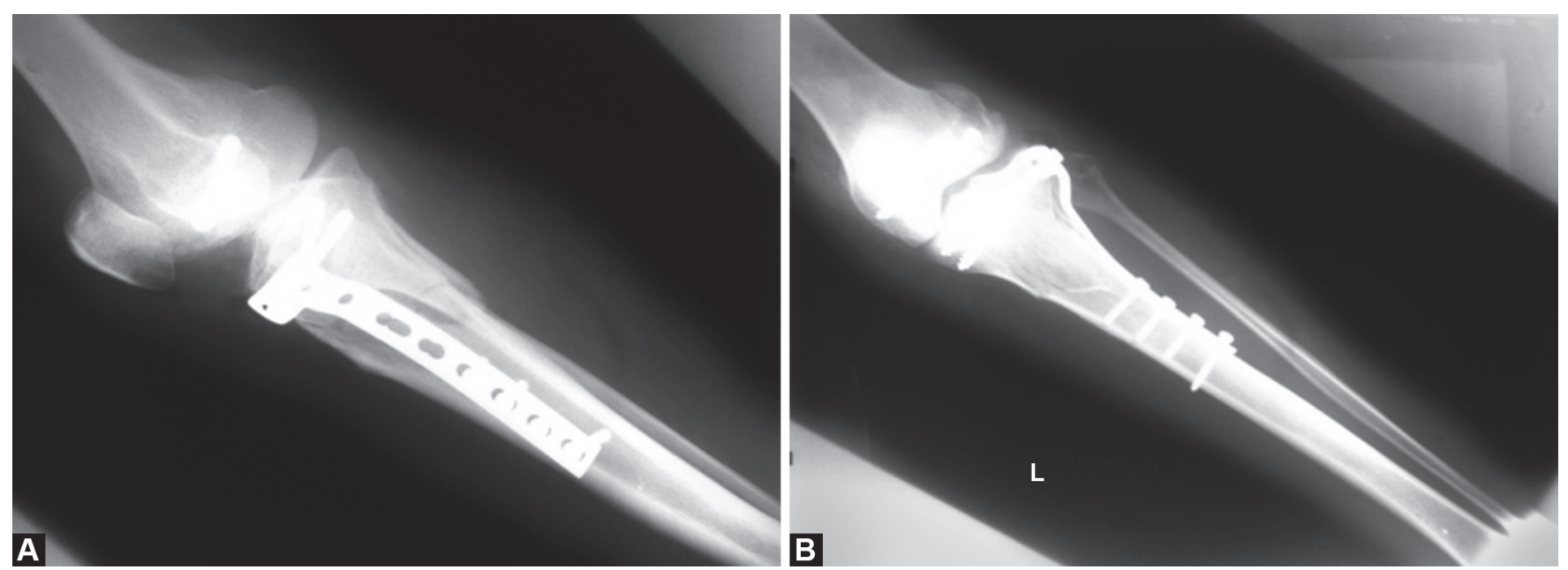

Figs $2 \mathrm{~A}$ and B: Postoperative X-ray

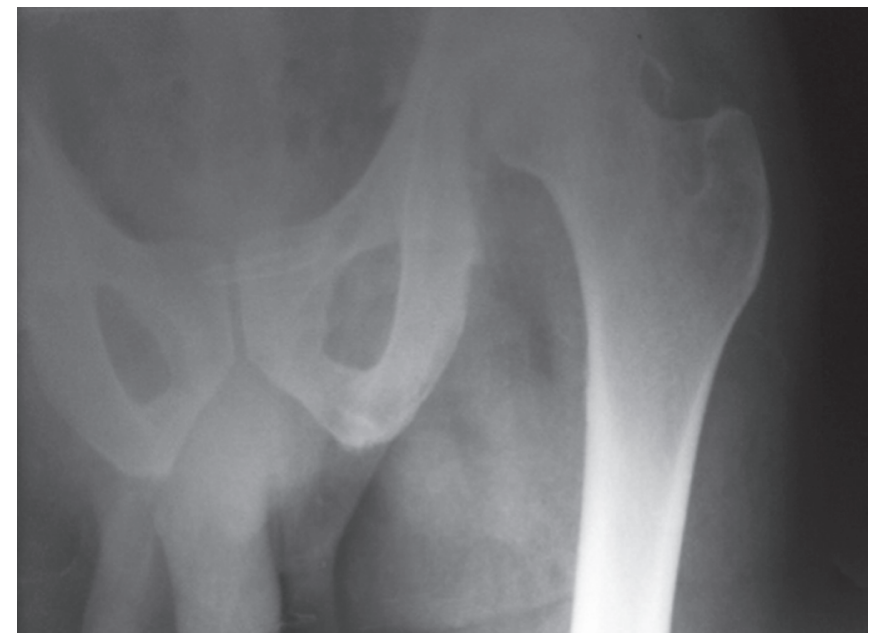

Fig. 3: X-ray showing posterior dislocation of the hip

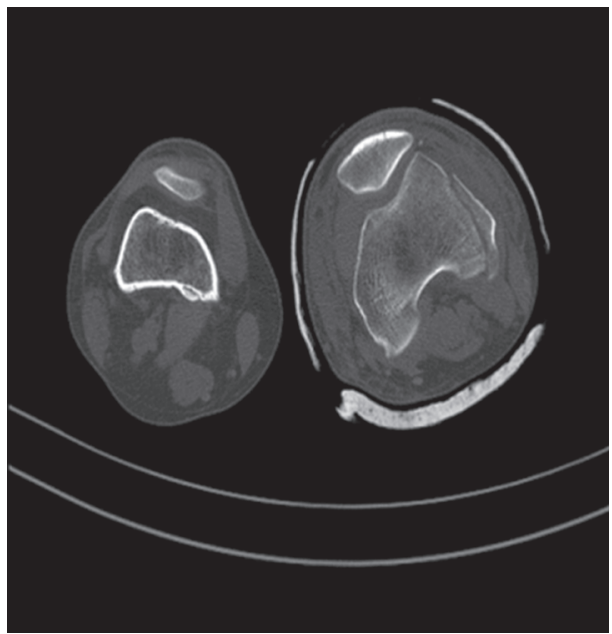

Fig. 5: CT scan showing internal rotation of the left thigh

until the twisting strain. As he came to us with above knee slab, we have missed the dislocation. Now, when we checked CT scan images again, we found the left patella facing medially and the limb was in internal rotation (Figs 4 and 5).

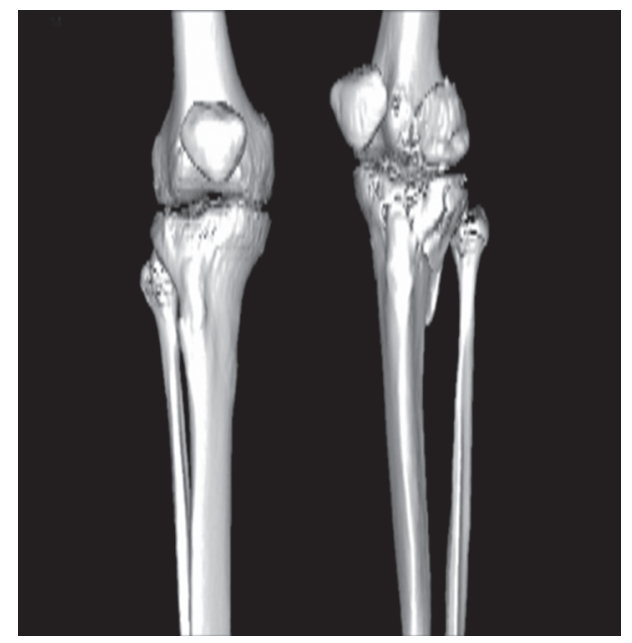

Fig. 4: CT scan showing internal rotation of the left thigh

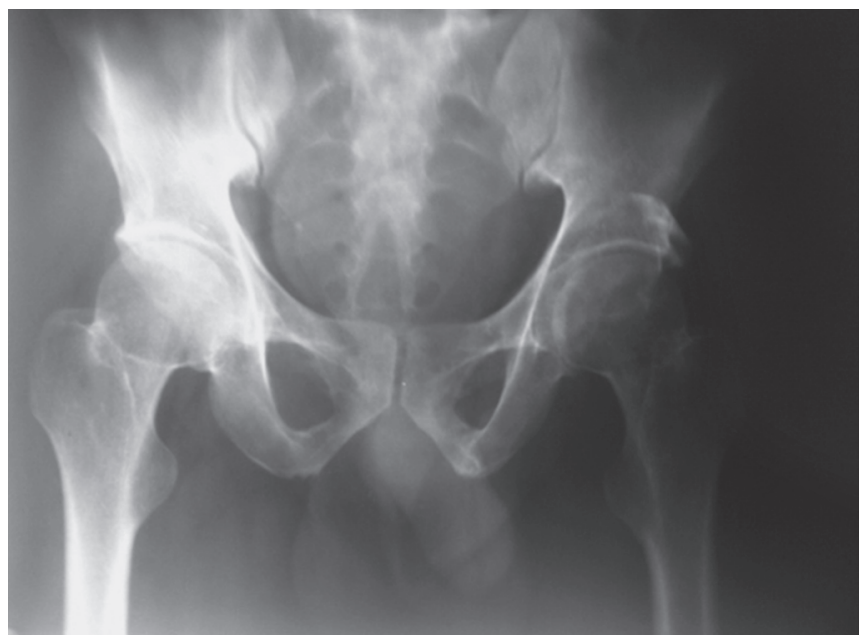

Fig. 6: X-ray showing concentric reduction of the left hip

There is no reason for the limb to be in internal rotation for upper tibial fractures. The dislocation was reduced under spinal anesthesia, and the reduction was confirmed in the X-ray (Fig. 6). 

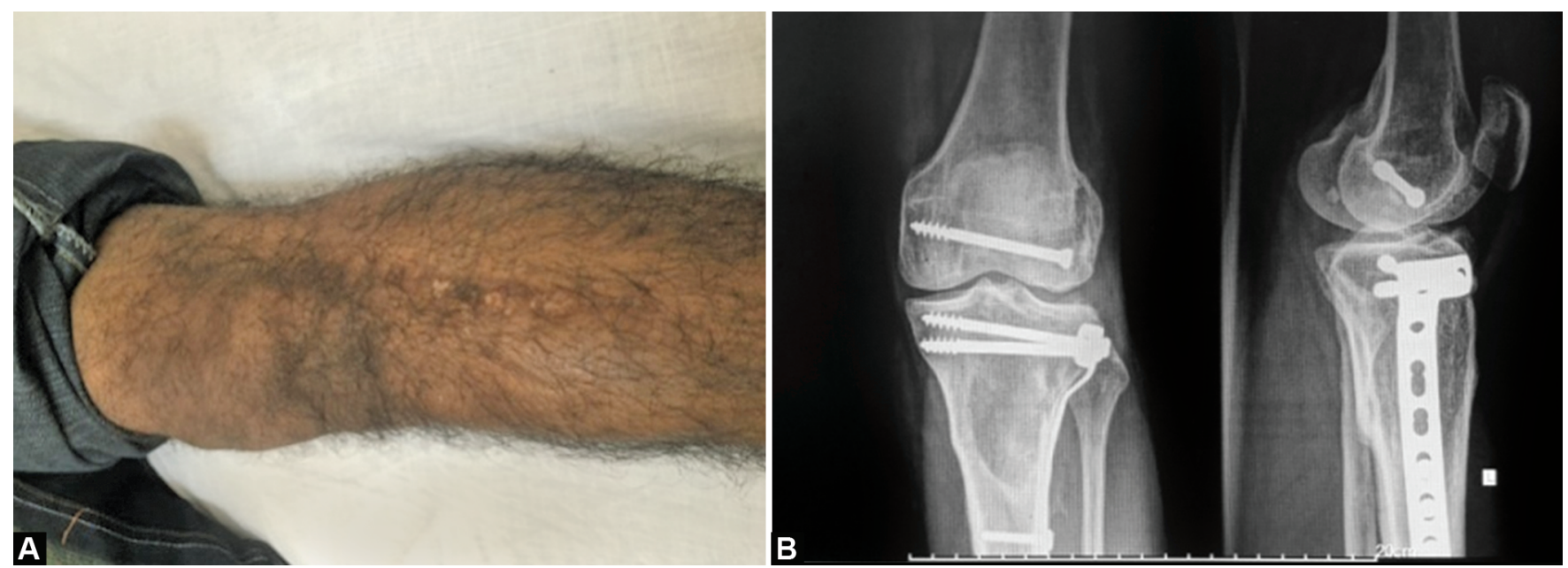

Figs 7A and B: Patient was kept in bed rest for 3 weeks, and later, mobilization of the hip and knee was done

The patient was kept in bed rest for 3 weeks, and later, mobilization of the hip and knee was done (Fig. 7).

\section{Discussion}

High index of suspicion of other injuries and especially in the joints is the need of the hour for those working in trauma and tertiary care centers where the patient may have multiple injuries, some of which may be the leading causes of mortality or morbidity. The same was the case in our scenario where a patient with much knee pain diagnosed as proximal tibia fracture was treated by us. Later, he was diagnosed to have dislocation of the hip which was missed by us in initial diagnosis. There are several other cases in the literature. Gillespie, ${ }^{3}$ in his article on "The incidence and pattern of knee injury associated with dislocation of the hip," a retrospective study of 135 posterior dislocations of the hip, has mentioned about the fracture of patella, fracture of femoral and tibial condyles, and other ligamentous injuries as associated injuries.

Hunter, ${ }^{4}$ in his article, "A review of 58 cases of posterior dislocation of the hip," has not mentioned about knee injuries (fracture of patella, bruising over the knee) accompanied with dislocation of the hip.
Hence, we are reporting this case of posterior hip dislocation as an associated injury of Schatzker type VI injury of the knee.

\section{Clinical Message}

Among the knee injuries, Schatzker type VI must also be included as one which can be associated with dislocation of the ipsilateral hip. So as the rule goes, X-rays of proximal and distal joints have always to be taken.

\section{References}

1. Mthethwa J, Chikate A. A review of the management of tibial plateau fractures. Musculoskelet Surg 2018;102(2):119-127. DOI: 10.1007/ s12306-017-0514-8.

2. Terry Canale S. Campbell Operative Orthopaedics. 11th ed., vol. 4. p. 3286.

3. Gillespie WJ. The incidence and pattern of knee injury associated with dislocation of the hip. J Bone Joint Surg Br 1975;57(3):376-378. DOI: 10.1302/0301-620X.57B3.376.

4. Hunter GA. Posterior dislocation and fracture dislocation of hip. J Bone Joint Surg Br 1969;51(1):38-44. 